Ronald B. Wilson

Roger F. Sles

U.S. DEPARTMENT OF COMMERCE Technology Administration National Institute of Standards and Technology Computer Systems Laboratory Office Systems Engineering Group Gaithersburg, MD 20899 



\title{
NEXT GENERATION DOCUMENTS
}

\author{
Ronald B. Wilson \\ Roger F. Sles
}

U.S. DEPARTMENT OF COMMERCE Technology Administration National Institute of Standards and Technology Computer Systems Laboratory Office Systems Engineering Group Gaithersburg, MD 20899

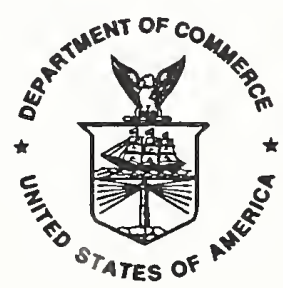

U.S. DEPARTMENT OF COMMERCE Barbara Hackman Franklin, Secretary

TECHNOLOGY ADMINISTRATION Robert M. White, Under Socretary for Technology

NATIONAL INSTITUTE OF STANDARDS AND TECHNOLOGY

John W. Lyons, Director 



\title{
NEXT GENERATION DOCUMENTS
}

\author{
by \\ Ronald B. Wilson \\ Roger F. Sies \\ Systems and Software Technology Division \\ Computer Systems Laboratory \\ National Institute of Standards and Technology
}

February 27, 1992

FY91 CALS TASK 2.1. "Define the goals for the next generation of documents."

"Develop an overall migration strategy."

"Sponsor small workshops with DoD and industry groups to discuss direction."

Deliverables

Report of the results of the workshops, goals and definition of next generation

documents, and preliminary migration strategy.

\section{- ABSTRACT .}

The object of this report is to identify the goals of the Next Generation Document (NGD). NIST sponsored two workshops on behalf of the Computer-aided Logistics and Acquisition Support (CALS) project. On July 30, 1990, a workshop on Electronic Information Exchange Standards Used in Document Processing Applications was held in an effort to bring together groups such as Standard Generalized Markup Language (SGML) and Open Document Architecture (ODA). On March 25, 1991, NIST presented a second workshop on Next Generation Documents (NGD). Staff members from various DoD services came together to exchange information on topics concerning next generation documents. These individuals were primarily supervisors working within the document processing field. NIST wanted to learn from them: 1) What is a next generation document, and 2) What requirements the next generation document must meet in the future. This report discusses the current DoD environment, its need to alter its business practices, and the movement towards the Open Systems Environment (OSE). The report also illustrates a NGD scenario and provides a listing of NGD requirements/services. 
- Table of Contents -

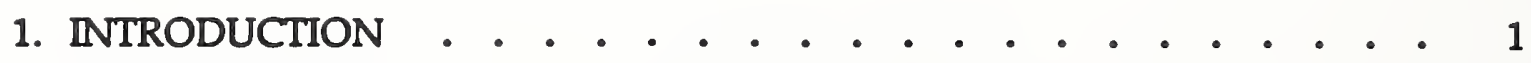

2. OPEN SYSTEMS ENVIRONMENT . . . . . . . . . . 2

3. NEXT GENERATION DOCUMENT SCENARIO . . . . . . . . . 5

4. WORKSHOP FINDINGS . . . . . . . . . . . . 9

5. CONCLUSION . . . . . . . . . . . . . . 12

6. BIBLIOGRAPHY . . . . . . . . . . . . . . 13

7. APPENDIX A . . . . . . . . . . . . . . 14 


\section{INTRODUCTION}

During the coming decade, the Department of Defense (DoD) will invest millions of dollars to develop "next generation documents." As part of this investment, the DoD will acquire a variety of computers and peripheral devices. The hardware will host a variety of operating system software and a suite of authoring system software in order to generate the wide variety of technical manual, memos, etc. needed in the day to day operation of the military. No single source of hardware or software systems will be available to satisfy the huge need. The DoD wide effort to establish standard software interfaces, along with the rest of the Federal government, is helping to cause a fundamental change in the information technology industry: the movement away from proprietary systems made up of proprietary products from a single vendor toward products based on open systems concepts. Currently, many systems are proprietary systems based on a single vendor's range of products. In the future, there will still be proprietary products in use within the information system, but the Open Systems Environment will allow for the exchange of information between many proprietary products.

The DoD is under increasing pressure due to the current budgetary crisis to use information technology to improve efficiency and effectiveness in the procurement of modern weapons systems. Therefore, the environment within the DoD must change. Before there were isolated islands of technology either within a particular group, division, or department. This type of situation would occur at a much higher level among major weapons systems prograrns. Each group or weapons system would have within it many duplicate efforts. These duplicate efforts would be necessary due to two factors: technology and politics. The technology was such that the various participants were unable to communicate with one another due to proprietary systems. The politics were such that each program director wanted complete control over their system with as little outside interference as possible. Due to the current fiscal environment as stated above, both these factors need to be addressed. There must now be an interdependence of users across an entire department or across a series of weapon system procurements. This interdependence highlights the need for common applications and system architectures, communication networks, and information bases. What is needed is an Open Systems Environment (OSE).

The open system movement is gaining momentum because it has become clear to many researchers and users that no single vendor can supply all the needs for our coming information based society. Open systems are needed to provide interoperability of products and portability of data and applications across heterogeneous computing environments. This same movement has spawned the creation of certain standards such as POSIX (Portable Operating System Interface for Computer Environments) and GOSIP (Government Open Systems Interconnection Profile). These two standards are a good start but fall short of addressing the full spectrum of future needs. 


\section{OPEN SYSTEMS ENVIRONMENT}

The Open System Environment (OSE) is a concept based on three precepts:

- Extensibility,

- Non-Proprietary, and

- Consensus-based.

Extensibility describes an architectural framework which allows an extensible collection of interfaces, services, protocols, and supporting formats to be defined. Non-proprietary describes interfaces, services, protocols, and supporting formats to be defined in terms of non-proprietary specifications that are available to any vendor for use in developing commercial products. Consensus-based describes an evolution that is controlled by a consensus-based process for decisions regarding definition and specification of interfaces, services, protocols, supporting formats, and other issues related to the computing environment. Services that would typically be included are user interface services, document management and interchange services, network services, operating system services, and document security services.

Technical goals of the Open System Environment may be characterized by the three items listed below:

- Portability,

- Interoperability, and

- Scalability.

Portability describes the ability to use application software and data on heterogeneous hardware/software platforms. Interoperability describes the ability to have application software operating on heterogeneous hardware/software platforms which cooperate in performing some user function. Scalability describes the ability to use the same application software on many different classes of hardware/software platforms ranging from personal computers to supercomputers.

Initially, the primary focus of portability was in applications. Later, it moved to operating systems. The need to improve portability led to standards such as POSIX and produced portable operating services and well defined programming interfaces. However, there was a major flaw in this approach. The operating system services and associated programming interfaces are elements of an OSE, but they do not provide sufficient functionality to meet the broad range of applications portability requirements. Additionally, the primary focus of interoperability centered on communication networks focusing on Open System Interconnection (OSI), network protocols, and data interchange formats. Again there was a flaw to this approach. Network protocols and data interchange formats are essential elements of an OSE, but they do not provide sufficient functionality to meet the broad range of requirements for applications/systems interoperability.

There is an emerging international consensus on the functionality (i.e., the collection of interfaces, protocols, services and supporting formats) that should be included in an OSE. There is no international agreement on the suite of specifications for the 
OSE functions. Organizations have used a variety of schemes to select their own specifications to define OSE functions. The result of these schemes are a suite of specifications called OSE profiles. The Applications Portability Profile (APP), developed at the National Institute of Standards and Technology, is an OSE Profile developed for use by U.S. federal agencies. The APP is defined in terms of open system specifications organized into major service categories.

Figure 1, page 4, depicts the OSE Framework or Reference Model. It shows the application level which would include document processing applications, courseware applications and many others. The services are shown on the middle level and are connected to the applications by the Application Program Interfaces (APIs). These APIs are interfaces from generic services to specific vendor application software products. A change in the application level would only require a new API. The data and the services would work with all specific application versions in this manner. The bottom level depicts the external environment platform. This would be interchangeable in much the same way as the applications, only using the Platform External Environment Interface instead of the APIs as the bridge. This model has been generalized to such a degree that it can accommodate a wide variety of general and special purpose systems. The OSE Reference Model is a set of concepts, interfaces, entities, and diagrams that provide a basis for specification of standards.

Application Software is defined as software specific to an application. It is composed of:

- Programs (e.g., source code listings, command files,);

- Data (e.g., user data, screen definitions, application parameters,); and,

- Documentation (e.g., on-line documentation,).

Separate but related standards supporting portability may be required for each of the components listed. While one or more applications may be run on a given platform simultaneously, each application can be thought of as an independent application entity, communicating with other applications.

The Application Platform is defined as the set of resources that support the services on which an application or application software will run. It provides services at its interfaces that make the specific characteristics of the platform transparent to the application. In order to assure system integrity and consistency, application software entities competing for application platform resources must access all resources via service requests across the API. Examples of application platform components could include an operating system kernel, a realtime monitor program, and all hardware and peripheral drivers. The platform might be a single processor shared by a group of applications, or it might be a large distributed system with each application dedicated to a single processor. This portion of the OSE will differ based on the size and requirements of the system and its intended use.

The External Environment comprises the external entities with which the application platform exchanges information. These external entities may include displays, disk drives, etc. Connecting the External Environment with the Application Platform is 


\section{The OSE Framework}

\section{DOCUMENT \\ PROCESSING \\ APPLICATION SOFTWARE COURSEWARE}

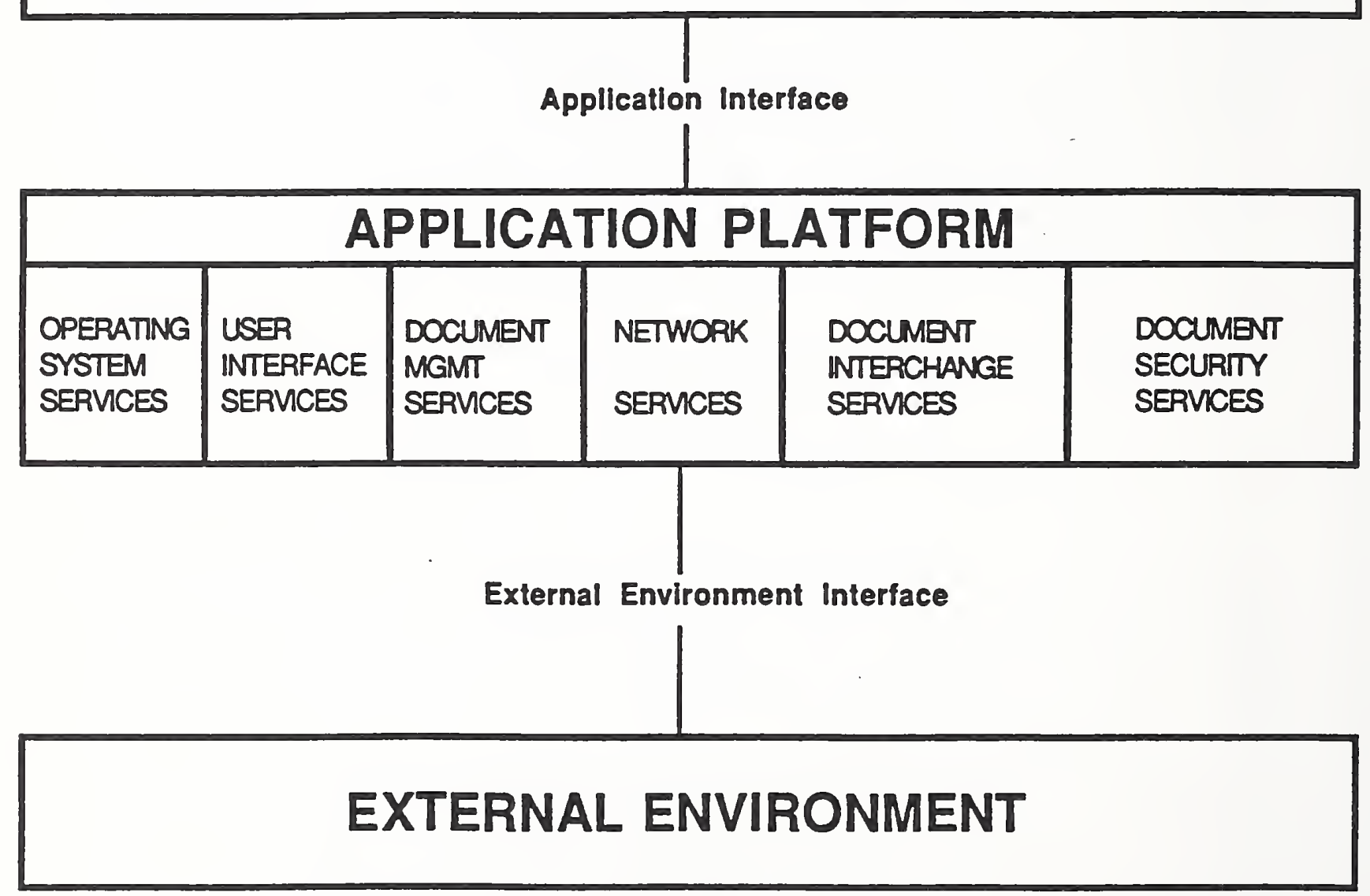


the External Environment Interface. The External Environment Interface is defined primarily in support of system and application interoperability. The primary services present at the External Environment Interface comprise:

- Human/Computer Interface - the boundary across which physical interaction between a human being and the application platform takes place;

- Information Interface - the boundary across which external, persistent storage service is provided; and,

- Communications Interface - the boundary between application software and the external environment, such as other application software, external data transport facilities, and devices.

\section{NEXT GENERATION DOCUMENT SCENARIO}

In the future, paper-based systems as we know them today will become large scale information-based open systems capable of electronically capturing text, graphics, images, engineering drawings, tables, mathematical equations, and art work. These systems will then be able to electronically store, manipulate, retrieve, and present the data in a variety of ways such as full motion video, animation, or paper-based documents. Users will be able to connect to the network from a multitude of locations and request many combinations of the data in a variety of formats.

Users would like to have all information sources available across all networks in real time. They want systems that overcome the barrier of multiple vendors, media, and locations. One major technological issue that must be resolved is the issue of version control. The placement of the data directly affects how version control will be implemented. Is it better for the data to reside near the author or near the user? There are advantages and disadvantages to both. If the data resides near the author, version control is much simpler. However, the distribution of the data becomes very difficult. With the data stored near its generation point, it can be updated in an accurate and timely manner. However, in the near term due to technology constraints, updating the data will have to be confined to a strict timetable. This will allow for adequate distribution of the revised data. If the data resides near the user, the issue of version control becomes much more difficult. Clearly, one of the major challenges ahead will be to effectively solve the dichotomy of single-point entry and multi-point access/distribution.

There is currently underway a rapid acceleration of information technology in a number of fields. One particular field which is often discussed is multimedia. Multimedia is a way of delivering a variety of types of information. Computermediated multimedia is the information delivery system of the future. The concept of multimedia includes notions currently popular such as hypermedia, computerbased training, simulation, image management, and image processing. Computermediated multimedia promises environments which will offer the users information, training, communication, production automation, and entertainment. Multimedia promises the delivery of multi-dimensional information based on the needs of the user via technologically efficient systems. These systems should be interactive, 
distributed computer systems operating in an open systems environment.

As an example, let's examine the role of someone who does repairs at a depot in the future open systems environment. This person is called upon to perform a corrective action. The first action that might be taken is to wheel out a terminal and connect up a cord from the terminal directly to the piece of hardware that has failed. There could be a connection on the hardware that can be used as a focal point for performing diagnostics on the individual components. After the connection has been made, the piece of hardware will inform the terminal of its model number and any other descriptive information indicating the identity of the hardware. After the terminal identifies the piece of equipment, it will search its index system so as to lead it to the database containing the document for that equipment. The program will present a series of options concerning that piece of equipment, which might include a diagnostic program or preventive maintenance program, etc. Each program could be represented by a graphic icon such as commonly used on various computers today. For this scenario, the repairperson could press the portion of the touch screen that displays a graphic of the diagnostic program. One of the first functions of the diagnostic program could be to query the hardware to determine what version of the equipment this is, i.e., what options this particular model contains. The next piece of information the terminal might present could be which diagnostics it is about to perform and provide a warning through the use of warnings, cautions, or notes about impending harm if in too close of contact with the hardware or certain parts. These warnings might not reside within each individual equipment's document, but may be retrieved from elsewhere through the network. (This capability would require some sort of linking service.) In performing this set of diagnostics, the program might resort to utilizing some sort of reasoning service, i.e., artificial intelligence.

This first operation could be performed in another way. The repairperson could carry a portable device, somewhat like today's portable computer, that is battery operated and contains its own information for that particular device. The repairperson could act as an intermediary in that the portable device could provide prompts to perform a series of procedures to determine which component has failed.

Either way is already in practice. The Air Force's Integrated Maintenance Information System Project should increase the efficiency of Base Maintenance operations by improving the effectiveness of technical information for base maintenance. It should display graphic technical instructions and intelligent diagnostic advice for aircraft base-level maintenance. Today's technology allows certain auto repair shops to connect the "black box" on the automobile to the diagnostic equipment allowing the equipment to perform failure analysis with no human intervention. As another example of today's technology, there are systems today in the medical field that provide a general practitioner with the knowledge of a specialist for diagnosing obscure diseases. Many of these systems are based on CD-ROM technology.

After the general diagnostics have been performed and the faulty component identified, the repairperson could remove the component and take it to the repair shop for more specialized corrective action. Once in the repair shop, a computer could be utilized in the same manner as discussed above. However, now the steps toward repair of the part would be more specialized. The repairperson could use the plug in method or act as the intermediary. In the near term, the person doing the repair would probably act as the intermediary and query the computer concerning 
the steps. The computer could then offer a touch screen or voice communication. Each step towards pin-pointing the failure could be represented on the screen as a graphic with a series of questions leading to the ultimate goal of diagnosing the failure. When it is time to take corrective action, a combination of graphics and text would serve best as the avenue of repair. There would have to be some sort of "consistency mechanism" to ensure the information supplied to the repairperson was accurate and the most updated version. This capability would be provided by an application and perhaps employ a reasoning service of its own.

During the repair, the system would certainly want to record the action taken. This would be necessary for a number of reasons. The system would need to update its supply of parts, and it would also be of value to "know" which parts have the highest mean time between failure. Other issues involved with this repair would include the amount of data the repairperson would be allowed to access. By stepping through a series of questions, the system could limit the amount of information accessed. Occasionally one might need to access information that was off-line and stored in a different physical location. This could be a background operation providing supplemental information offering additional insight. This type of operation could be utilized in other processes within this scenario.

In accessing the information, other issues would need to be addressed such as was this the most up-to-date information. The system would have to deal with legacy data while ensuring the information it supplied was correct. Additionally, the system would have to be designed with in-depth quality control features and a method for providing the user with the most recent version of the data. The system might also want to add the capability of allowing the repairperson to offer helpful insights or "tips" for performing the operation. However, this feature would require review at higher levels to ensure these "tips" were no more than a dangerous shortcut in performing the corrective action. Adding helpful tips could be a very fruitful capability in the long run. We know that each generation of human beings is required to re-learn many day-to-day procedures. If we had a better capability of imparting learned information from one generation to the next, our productivity would be greatly increased. We do this to varying degrees today through the use of books. In a sense, many military systems do this today by incorporating previous experiences into the repair steps since some of these repairs have been ongoing for many years. However, one wonders for the few changes that are recorded how many more go unnoticed and forgotten. There is no doubt that this accumulation of knowledge would be greatly accelerated with an advanced system as described here.

The user interface of this repair system should be such that one would not have to be an expert with the system to use it. For example, it could be similar to an automobile where one is not required to be familiar with each make of automobile, but with very little effort, one can get into a different model and in a brief period of time operate it. Within the Open Systems Environment Model (Figure 1, page 4), the user interface would be contained at the application level thereby not requiring the platform level to be different for each system. Also, to the user, the application would look similar irrespective of which platform was being used.

We realize today that the complexity of the weapons systems is growing at an astounding rate. The amount of material that is necessary for performing corrective actions on these systems is also growing. Therefore, the amount of knowledge that 
must be accumulated and stored to support each of these systems is growing at an alarming rate. One may argue that the problem is not the amount of information that must be managed, but the method of accessing the information and the method of ordering the material. Technology will provide the means to reduce storage requirements for this data while increasing the retrieval rates of the data. Additionally, a redesign of the methodologies in use for supplying the information to the people in the field must be performed. As system designers there are three avenues available. We can:

1. look at what can be,

2. look at what should be, or

3. look at what will be.

It is clear from past experience that if nothing is done, we will emulate the past. In effect, the system design will be driven by the legacy data. Alternatively, the system design can be driven by the requirements of a new weapon system. This would lead us down the same path we have taken before: each information system would be designed specifically for its own weapon system not allowing the government to realize any economies of scale such as interchange of hardware platforms and information type. Clearly, the path to choose involves looking at what can be and utilizing today's technologies while incorporating tomorrow's emerging technologies, whether they are currently conceived or envisioned. Only in this manner may the government realize large scale interchangeability of hardware and software, thus enabling it to dramatically reduce future costs while providing greater functionality to the field.

In order for the government to realize the needed economies of scale due to today's economic and political environment, it must turn it's attention to the open systems movement. The Next Generation Document is an ideal starting point for this process to begin. Open Systems are built on standards that define application and system interfaces in:

- Operating Systems;

- User Interfaces;

- Data Management;

- Networking; and

- Graphics and Software Development.

As we have seen from the above scenarios, Next Generation Documents will also utilize applications and system interfaces in these same areas. 


\section{WORKSHOP FINDINGS}

On July 30, 1990, the Office Systems Engineering Group at NIST presented a workshop on Electronic Information Exchange Standards Used in Document Processing Applications. The workshop was sponsored by the Computer-aided Acquisition and Logistics Support (CALS) project. The workshop was one of our efforts to bring the various factions such as Standard Generalized Markup Language (SGML) and Open Document Architecture (ODA) together. The second goal of this workshop was to develop a set of user requirements for electronic information exchange standards and document processing applications. The workshop provided a forum for individuals in private industry and government to exchange information on topics that relate to the selection and application of electronic information exchange standards such as ODA, SGML, Document Style Semantics and Specifications Language (DSSSL) and Standard Page Description Language (SPDL), among others, that are used in document processing environments.

During the course of the workshop, a few issues emerged as being of primary importance to a number of the speakers. The primary requirement articulated by the majority of the speakers was the need to move toward information management in a database environment rather that through separate documents. In conjunction with this requirement was a second strong requirement to have a migration strategy that considers legacy data. There were many other requirements presented which are listed in the "Gooernment Document Processing Requirements Report," NISTIR 4560.

Many of the requirements identified were illustrated in the previous section of this paper. Some examples are:

- Ability to encode the information itself to provide an information service independent of the application/device;

- On-line display;

- Query and retrieval ability;

- Electronic deliverables and pageless technical manuals;

- Simplified control over document creation and management in a distributed environment; and,

- Ability to insure correctness while using digital media.

On March 25, 1991, the Office Systems Engineering Group at NIST presented a workshop on Next Generation Documents (NGD). The workshop was sponsored by the Computer-aided Acquisition and Logistics Support (CALS) project. Government individuals from the different services came together to exchange information on various topics concerning next generation documents. These individuals were primarily supervisors working within the document processing field. The list of 
attendees are listed in Appendix A. NIST wanted to learn from them:

- What is a next generation document, and

- What requirements the next generation document must meet in the future. How will they be used, (i.e., stored, retrieved, accessed, version control,).

The workshop attendees believed that lessons could be learned from previous weapons systems procurements. They stated it was necessary to allow for portability of information products across various hardware platforms, and that Next Generation Document standards needed to be developed to capitalize on the manner in which large documents or document sets are developed and ultimately used. They believed that while individual weapons systems are different, many have similar information requirements that Next Generation Documents (NGD) could help standardize. Technical manuals were an example of a class of documents where registered NGD application models could be developed.

The participants expressed their views as to the future of document processing and a list of requirements/services was established from this workshop. Services within OSE can be divided in the following manner:

1. Document Management Services

- Data Dictionary/Directory (Baseline Tag Set and meanings)

- Information Management System

- Distributed Information

- Information Management Security

2. Diagnostics Services

- Preventive Maintenance Procedures

- Interactive Procedures

- Cautions, Warnings, and Notes to Repair Personnel

- Automated Test Procedures

- Record Keeping of Maintenance

- Reasoning Procedures

- Expert Systems Procedures

- Controlled Dynamic Additions and Changes to Diagnostics

3. Configuration Services

- To keep track of all the different relationships data can have to itself.

4. Archival Services

- Back-up of systems. 
5. Consistency Mechanism Services

- Ensure that all information is internally consistent.

- Ensure data version control.

- Ensure that all information is complete.

6. User Interface Services

- Graphical Interface

- Touch Screen

- Voice Commands and Communications

7. Document Security Services

8. Document Interchange Service

- Must be able to interchange not only data, but the relationship and attributes of the data.

9. Data Services

- Data Capture - text, graphics, images, engineering drawings, tables, mathematical equations, and art work.

- Storage

- Data Search and Retrieval

- Linking to Remote Data

10. Other Services

- Presentation

- Networking

- Formatting

- Realtime Updating

- Image Management

- Image Processing

- Full Motion Video

- Animation

- Document Processing

- Weapon Systems Statistics Collection and Presentation

- System Security

Document Management Services consist of Data Dictionaries developed for the various weapons systems or the Baseline Tag Set contained within MIL-M-28001A, Markup Requirements and Generic Style Specification For Electronic Printed Output and Exchange of Text. This Baseline Tag Set must be continually managed, which involves updating the tags (version control) and distributing the information in a timely manner to ensure its usefulness. 
Diagnostic Services are concerned with providing the services as described in the scenarios listed in the previous section. This group of services contains maintenance procedures that are automated, real-time, interactive, and/or expert type systems. Adequate records must be maintained and allowed to become a dynamic library.

Consistency Mechanism Services are of the utmost importance since all information must be up-to-date to ensure adequate repair facilities and deter against accidents. The issue of version control has been addressed many times within this paper and presents one of the critical technological issues that the Next Generation Document must resolve to be successful.

User Interface Services as listed above are already in use in selected applications. The Graphical Interface is in use in a multitude of computer systems today and with the introduction of Windows on DOS systems, it appears a large percentage of the industry is going in this direction. Touch Screens are also in use today but in a much more limited scope than the graphical user interface. Voice Communications are still in somewhat of an experimental stage. This interface is being used on an extremely limited basis for a limited audience.

\section{CONCLUSION}

In examining the results of the two workshops, it becomes clear that many of the concerns expressed within the first workshop were echoed by the participants at the second workshop. In order for the government to meet the future requirements stated within this document, it must now turn its attention away from single vendor proprietary systems and commit itself to the Open Systems Environment. It is inevitable that proprietary products will always exist, so a framework must be established to allow for intelligent communication between these products. It is clear that future requirements can never be completely described since technology is constantly changing. And as the technology changes, so will the functional desires of each organization. Therefore, the intelligent migration strategy for Next Generation Documents is to utilize a framework based on open specifications for interfaces, services, supporting formats, etc. The Open Systems Environment concept based on extensibility, consensus-based, and non-proprietary systems with technical goals of portability, interoperability, and scalability appears to be the clear path toward a successful implementation of Next Generation Documents. 


\section{BIBLIOGRAPHY}

[1] IS 8879: Information Processing - Text and Office Systems - Standard Generalized Markup Language (SGML), International Organization for Standardization, 1986.

[2] Document Application Profile (DAP) for the Office Document Architecture (ODA) and Interchange Format Standard, National Institute of Standards and Technology, December 1988.

[3] IS 8613: Information Processing - Text and Office Systems - Office Document Architecture and Interchange Format (ODA). International Organization for Standardization, 1989.

[4] MIL-M-28001A - Technical Manuals: Markup Requirements and Generic Style Specification for Electronic Printed Output and Exchange of Text, July 1990.

[5] TSO work item number JTC1.18.15.06.01, Information Processing - Text Composition - Document Style Semantics and Specification Language (DSSSL)."

[6] Roger F. Sies, Government Document Processing Report, NISTIR 4560, April 1991.

[7] "APPLICATION PORTABILITY PROFILE (APP) The U.S. Government's Open System Environment Profile OSE/1 Version 1.0," National Institute of Standards and Technology, April 1991.

[8] "ISO/IEC DIS 10180, Information Processing - Text Communication - Standard Page Description Language (SPDL), International Organization for Standarization, 1991. 


\section{APPENDIX A}

The following is the list of attendees at the March 25, 1991 workshop on Next Generation Documents.

\begin{tabular}{|l|l|l|}
\hline \multicolumn{1}{|c|}{ Attendee } & Organization & Phone Number \\
\hline John Winters & MMD/TDD & $(703) 756-2554$ \\
\hline Joe Fuller & DTRC & $(301) 227-1358$ \\
\hline Eric Jorgensen & DTRC & $(301) 227-1622$ \\
\hline Lt. Brian Caporlette & AFHRL/LR & $(513) 255-2606$ \\
\hline Ivan Galysh & HQ-USAMC & $(703) 274-9151$ \\
\hline Susan Brookins & JUSTIS & $(703) 756-0996$ \\
\hline Gus Apsitis & MRSA & $(606) 293-3415$ \\
\hline Judi Brisson & MRSA & $(606) 293-3415$ \\
\hline Joe Rogowski & HQ-CECOM & $(908) 532-1939$ \\
\hline William Campbell & MICOM & $(205) 876-9513$ \\
\hline Hope Robinson & APPS & $(703) 325-6255$ \\
\hline Maj. McAvoy & APPS & $(703) 325-6255$ \\
\hline Lt. Col. Jim Stoucker & AF902S EPPO & $(703) 746-6956$ \\
\hline Phil Clark & LMI & $(301) 320-7310$ \\
\hline
\end{tabular}


NIST-114A

(REV. 3-0)

\section{U.S. DEPARTMENT OF COMMERCE \\ NATIONAL INSTITUTE OF STANDARDS AND TECHNOLOGY BIBLIOGRAPHIC DATA SHEET}

1. FUUUCATION OR REPORT NUMEER NISTIR 4830.

2 PERFORMINO ORCANIZATION AEPOAT NUMBEA

3. PUEUCATION DATE

APRIL 1992

4. TITE AMD SUDTITLE

NEXT GENERATION DOCUMENTS

5. AUTHOA(S)

Ronald Wilson

6. PERFORLIMO ORCAMIZATION (IF JOINT OA OTMER THAN MIST, SEE WSTRUCTIONS)

U.S. DEPARTMENT OF COMMEACE

MATIONAL IASTITUTE OF STANDARDS AMD TECHMOLOQY

CATMERSBURG, MD 20090

7. CONTRACT/ORANT MUMEER

Q. TYPE OF REPORT AND PEAIOD COVERED

9. SPONSOAING ORGANIZATION MANE AND COMPLTE ADDAESS (STRET, GT, STATE, ZIP)

NIST/CSL

Division 872

Gaithersburg, Maryland 20899

10. SUPPLEMENTARY MOTES

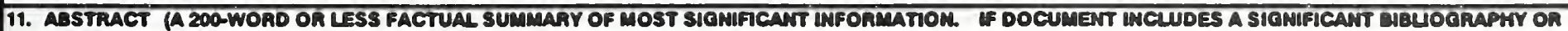
UTERATURE SURVEV, MENTION IT MERE)

On March 25, 199: the Office Systems Engineering (OSE) Group at the National Institute of Standards and Technology (NIST) presented a workshop on Next Generation Documents (NGD). The workshop was sponsored by the Computer-aided Acquisition and Logistics Support (CALS) project. Government individuals from the different services came together to exchange information on various topics concerning next generation documents. Theses individuals weré primarily supervisors working within the document processing field. NIST wanted to learn from them: 1) What is a next generation document, and 2) What requirements the next generation document must meet in the future, and how will they be used (i.e., stored, retrieved, accessed, version control).

The workshop attendees believed that lessons could be learned from previous weapons systems procurements. They stated it was necessary to allow for portability of information products across various hardware platforms, and the Next Generation Document standards needed to be developed to capitalize on the manner in which large documents or document sets are developed and ultimately used.

12. KEY WORDS (6 TO 12 ENTRIES; ALPHABETICAL ORDER; CAPTTALIE ONLY PAOPER MAMES; AND SEPARATE KEY WORDS BY SEMICOLOMS)

Next Generation Documents, CALS, Open Systems Environment, P0SIX, G0SIP, MIL-M-28001A

FOR OFFTCIAL DISTAIBUTION. DO NOT REEASE TO NATIOMAL TECHMICAL INFOAMATION SEAVICE (NTIS).

ORDER FROM SUPERINTENDENT OF DOCUMENTS, U.S. COVERMMENT PRINTING OFFICE, WASHINOTON, DC 20402.

ORDER FROM MATIONAL TECHMICAL IMFORMATION SEAVCE (NTIS), SPRINOFIED, VA 22161.

14. NUMBER OF PRINTED PAGES

19.

15. PAICE

$\mathrm{A} 02$ 

